\title{
UTILISATION OF HEALTH MANAGEMENT INFORMATION SYSTEM IN DISEASE SURVEILLANCE IN TANZANIA
}

\author{
L.E.G. Mboera, S.F. Rumisha, S.M. Magesa E A.Y. Kitua \\ National Institute for Medical Research, \\ P.O. Box 9653 , \\ Dar es Salaam, Tanzania
}

\begin{abstract}
The objective of this study was to assess the utilisation of the current health management information system (HMIS) in disease surveillance and response in selected districts of Tanzania in 1999. The findings of this assessment has shown that between $17.8 \%(8 / 45)$ and $44.4 \%(20 / 45)$ of the districts could not provide demographic data on infant mortality, under five mortality and maternal mortality rates. In $51.1 \%$ of the districts, data collection, analysis and utilisation were poor. Only $15.6 \%$ (7/45) of the districts used the data collected for surveillance purposes. Over $50 \%$ of the districts reported outbreaks of measles, meningitis, cholera and dysentery. Districts that utilised health information for disease surveillance had a low number of epidemics and only $34.7 \%$ of them reported disease outbreaks. In order to improve the HMIS, the Tanzanian Ministry of Health has adopted an integrated disease surveillance and response strategy. However, if this strategy is to be successfully in reducing morbidity and mortality, it must ensure timely collection and utilisation of disease surveillance information at district level.
\end{abstract}

\section{Introduction}

Communicable diseases continue to cause most of the morbidity and mortality in Tanzania (1). The commonest causes of morbidity and mortality in Tanzania include malaria, upper respiratory tract infections, diarrhoeal diseases, and pneumonia. Epidemic prone diseases such as cholera, measles, meningitis, plague, typhoid fever, and dysentery are becoming more important today than ever before. Some of the reasons which may lead to the increased number of those diseases include reduced immunisation coverage, competing priority for resources, increased transmission risks due to migration from rural to urban areas, poverty, overcrowding, poor hygiene (2) and weakness in our surveillance system (3).

The Tanzanian Ministry of Health maintains two major systems for collecting and analysing information regarding infectious diseases, the Health Management Information System (HMIS) also known by its Kiswahili acronym MTUHA (Mfumo wa Taarifa za Uendeshaji Huduma za Afya), and the Infectious Disease Week Ending Report (IDWE). The HMIS was introduced in Tanzania between 1993 and 1997 to replace the old system known as the Health Information System (HIS). The HMIS was introduced due to the fact that the former HIS was inadequate and inappropriate to the growing information needs of the health sector. The former system was also fragmented in nature with bottom-up flow of information and with little or no feedback to the lower levels. There was also little capacity for analysis. interpretation and use of the data at various levels of the health care delivery system (4).

In preparation for the on-going health sector reform, it was necessary to carry out a situation analysis of the health delivery system in districts of Tanzania so as to provide baseline information for future district health plans. In this paper the current health management information system and its utilisation in disease surveillance and response is discussed.

\section{Materials and Methods}

An assessment of the health management information system was carried in 45 randomly selected districts of Tanzania. The districts under the assessment included Arumeru, Bagamoyo, Bukoba rural, Bunda, Dodoma rural, Geita, Handeni, Igunga, Kahama, Kibaha, Kibondo, Kigoma urban, Kilosa, Kwimba, Kyela, Lindi rural, Lushoto, Makete, Manyoni, Maswa, Mbeya rural, Mbozi, Mbulu, Meatu, Morogoro rural, Mpanda, Mtwara rural, Mufindi, Muheza, Muleba, Nachingwea, Newala, Ngara, Rombo, Ruangwa, Rufiji, Same, Sengerema, Serengeti, Singida urban, Songea rural, Sumbawanga urban, Tandahimba, Tabora rural and Tunduru. The methodology used in collection of data is described elsewhere (2). The findings reported in this paper cover analyses of data on demographic indices and communicable diseases for the year 1999.

\section{Results}

Lack of information on current demographic parameters and disease patterns was observed in most districts (Table 1). Follow-up of health information collection, analysis and reporting was poor at district level. Detailed analysis of district reports and district processing files for 1999 showed that only $20 \%$ (9/45) of the districts were able to present a full range of demographic indices. $37.8 \%, 44.4 \%, 17.8 \%, 37.8 \%$ and $44.4 \%$ of all districts had no records on infant mortality, under 5 year mortality, maternal mortality, crude birth and fertility rates respectively (Table 1). It was observed that different 
definitions and calculation procedures were used for both reproductive and child health indicators by the districts.

As high as $51.1 \%(23 / 45)$ of the districts collected and compiled health data without knowing their use. Only $15.6 \%(7 / 45)$ of the districts used the data collected fo surveillance purposes. The rest of the districts $(33.3 \%)$ used the data for setting their annual health plans (Figure 1). Urban-rural comparison showed that none of the urban surveyed districts utilised the collected data for surveillance purposes.

Of the 23 districts not utilising health information for planning or surveillance, $43.5 \%$ reported at least 3 disease outbreaks in 1999. Of the districts that claimed to utilise health data for planning and decision-making, $48.6 \%$ reported at least 4 outbreaks of important epidemic-prone diseases. Districts that utilised health information for disease surveillance had a low proportion of epidemics (an average of 2 diseases per district) and only $34.7 \%$ of them reported outbreaks (Table 2 ).

Over $50 \%$ of the districts reported epidemics due to cholera, dysentery, measles and meningitis in 1999. Although epidemics were reported in most districts, no efforts were made to monitor any annual increase in number of cases to contain future outbreaks (Table 3).

\section{Discussion}

Our findings have shown gross lack of information on current demographic parameters and disease patterns in the districts. Generally, most districts of Tanzania have indicated difficulties in obtaining demographic indices. The main reason for lack of important demographic data in the districts is most probably due to general poor data management. This may be explained partly to be due to low knowledge of DHMTs on calculating simple demographic statistics and other indices.

The number of districts that utilise health information for disease surveillance is quite small. Interestingly these are districts that experience few disease epidemics as compared to districts that claim to use the information for planning and decision-making. It is realised in this study that the biggest problem in our public health system is the lack of surveillance mechanism that should help districts to monitor epidemic prone diseases and other diseases of public health importance. It is already known that a well designed and well-implemented surveillance programme can provide means to detect epidemics early, document their geographical and demographic spread, estimate the magnitude of the outbreak and initiate timely action (5). It is most likely that the lack of an efficient surveillance system has left policy makers with no basis for developing and implementing policies for controlling the spread of epidemic prone dis ases.

A further analysis of the current disease surveillance systems has shown that in most districts there is shortage of data processing tools, over-burdened health staff, weak communication systems, lack of strong laboratory facilities for confirming diagnosis and weak mechanism for synergistic action in the area of epidemic preparedness and control. The presence of multiple surveillance systems in the country demanded by different vertical programmes results in duplicity and inefficient use of resources.

Although, to-date HMIS is available throughout the country, health data collection, analysis, reporting and utilisation in disease surveillance is poor. The recently introduced integrated disease surveillance (IDS) and response strategy by the Ministry of Health is a way forward to the improvement of our health management information system. The country has recognized the need for improving its disease surveillance system and identified IDS strategy as the best approach.

There are several opportunities, which make the introduction of a new approach for disease surveillance timely. These include: the ongoing health sector reforms demanding the decentralization of action and decision to district level; the necessity for strengthening capacities for data collection, analysis and prompt utilization; the need for good quality and reliable information for planning at district level; the new establishment of Sector Wide Approach in the allocation of resources in the health sector; the provision of additional funds through the basket funding mechanism whereby money will be directed at national priorities; and the awareness of disease surveillance problems and country demand to improve the situation.

In conclusion, effective disease control requires prompt and adequate action towards reduction or elimination of existing conditions, and preventing the occurring of new ones. Such actions can only be made if correct information reaches those required to take action timely. It is therefore essential to strengthen disease surveillance systems at all levels so that data collected is correct and credible, analyzed adequately and promptly, and passed on for action timely. Such a system must also be efficient, especially when it concerns countries with limited resources such as Tanzania.

\section{Acknowledgements}

We are grateful to the two anonymous reviewers of the early draft of the manuscript. 


\section{References}

1. Mwisongo, A., Mboera, L.E.G., Alilio, M., Kabali, C., Kisoka, W., Kamugisha, M., Mutalemwa, P., Msangi, J., Laswai, C., Malecela, M. \& Kitua, A. (2000) Situation Analysis of Districts under Phase I Health Sector Reforms. Report Submitted to the Ministry of Health. Dar es Salaam, Tanzania, March 2000.

2. Magesa, S.M., Mboera, L.E.G., Rumisha, S.F. \& Kitua, A.Y. (2001) Major Health problems in some selected districts of Tanzania. Tanzania Health Research Bulletin (in press).

3. Mboera, L.E.G., Rumisha, S.F. \& Kitua, A.Y. (2001) Strategic approach for strengthening national and regional disease surveillance systems: The East African example. Tanzania Health Research Bulletin (in press).

4. Mwangu, M.A. \& Otito, C.K. (2000) MTUHA implementation at the district level in the context of the health sector reforms: Challenges and future prospects. Sector Reforms and Health in Tanzania. Proc. $18^{\text {th }}$ Annual Scientific Conference of the Tanzania Public Health Association, Nov. 22-25, 1999, CCT Conference Centre, Dodoma, pp.111-119.

5. Dagnew, M., Fantahun, M. \& Ismail, S. (1995) Epidemic prone diseases in Ethiopia. Ethiopian Journal of Health Development 9, 118-130.

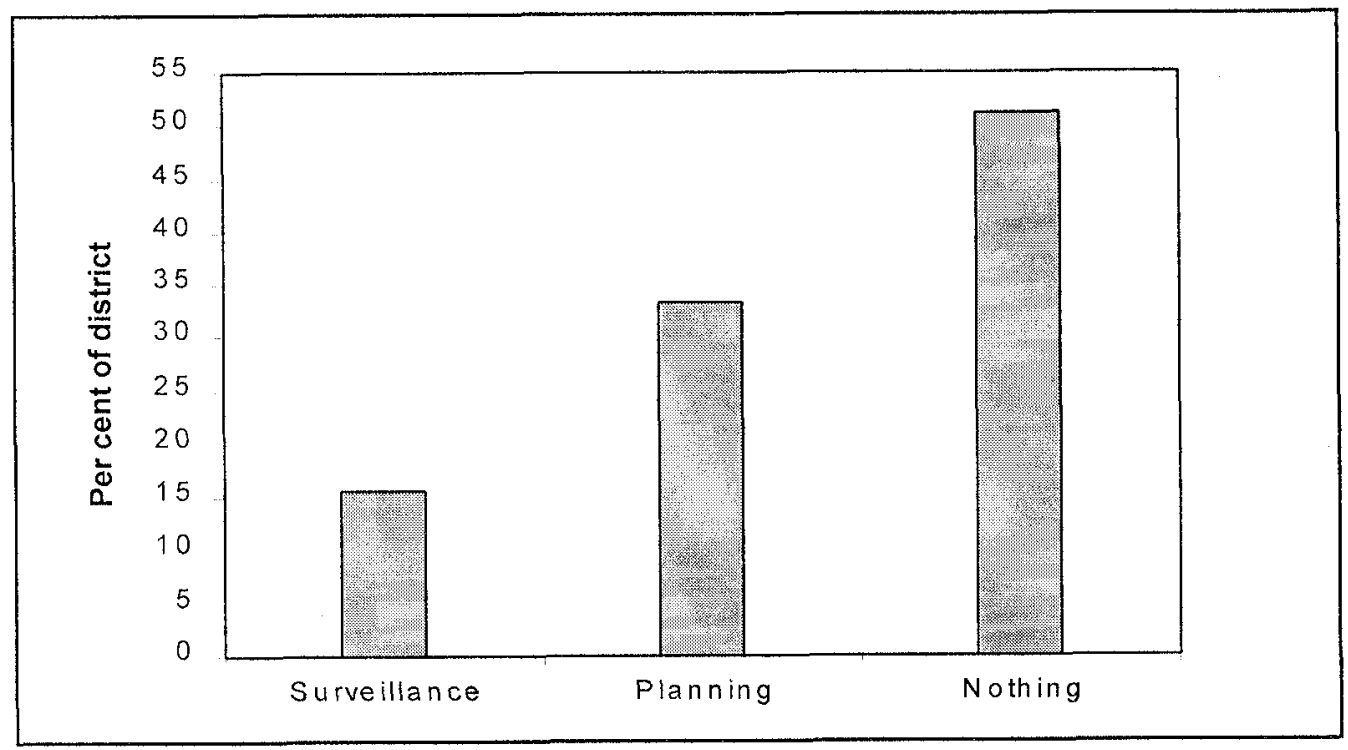

Figure 1: Utilisation Pattern of Health Data in Selected Districts of Tanzania

Table 1: The Proportion (\%) of Districts not Reporting Basic Demographic Indicatiors in Tanzania

\begin{tabular}{|l|c|}
\hline Demographic Indicator & $\%$ \\
\hline Infant mortality rate & 37.8 \\
Under five mortality rate & 44.4 \\
Maternal mortality rate & 17.8 \\
Crude birth rate & 37.8 \\
Fertility rate & 44.4 \\
\hline
\end{tabular}

Table 2: Percentage of Districts Reporting Disease Outbreaks in Relation to Utilisation of Health Management Information System in Tanzania

\begin{tabular}{|l|c|c|c|}
\hline Disease & Planning \& Decision Making & Disease Surveillance & $\begin{array}{c}\text { Neither for Planning nor } \\
\text { Surveillance }\end{array}$ \\
\hline Cholera & 53.0 & 42.8 & 66.7 \\
\hline Measies & 60.0 & 28.6 & 42.8 \\
\hline Typhoid fever & 60.0 & 28.6 & 42.8 \\
\hline Acute flaccid paralysis & 20.0 & 0 & 28.6 \\
\hline Neonatal tetanus & 0 & 28.6 & 28.6 \\
\hline Meningitis & 73.3 & 57.1 & 47.6 \\
\hline Dysentery & 73.3 & 57.1 & 47.6 \\
\hline Average & 48.6 & 34.7 & 43.5 \\
\hline
\end{tabular}


Table 3: The Percentage of Districts Reporting Disease Outbreaks in 1999

\begin{tabular}{|l|c|}
\hline Disease & Percent of Distict \\
\hline Cholera & 64.4 \\
Meningitis & 55.6 \\
Plague & 6.70 \\
Rabies & 33.3 \\
Dysentery & 62.2 \\
Measles & 53.3 \\
Typhoid fever & 48.9 \\
Acute flaccid paralysis & 24.4 \\
Neonatal tetanus & 17.8 \\
\hline
\end{tabular}

